\title{
Phase Behavior and Critical Properties of Size-Asymmetric, Primitive-Model Electrolytes
}

\author{
Yuan Qin and John M. Prausnitz \\ Department of Chemical Engineering, University of California, Berkeley, and Chemical Sciences \\ Division, Lawrence Berkeley National Laboratory, Berkeley, CA 94720
}

\begin{abstract}
The theory of J. Jiang et al. [J. Chem. Phys. 116, 7977 (2002)] for size-symmetric electrolytes is extended to size-asymmetric electrolytes. When compared to molecular-simulation results, this extension gives the correct trend of critical properties with size asymmetry.
\end{abstract}

\section{Introduction}

Debye-Hückel theory ${ }^{[1]}$ and the mean-spherical approximation (MSA) theory ${ }^{[2]}$ provide two fundamental analytical theories for calculating the equilibrium properties of electrolytes. However, because both are linearized theories, they are restricted to large reduced temperatures where the kinetic energy is large compared to the electrostatic energy. At low reduced temperatures, positive ions and negative ions tend to form clusters; therefore, Bjerrum's ion-association concept is useful for improving a linearized electrolyte theory. While the definition of ion-association has some intrinsic arbitrariness, Ebeling ${ }^{[3]}$ has given a definition of the ion-association constant that yields the correct equation of state to the second-ionic-virial-coefficient term. Gillan ${ }^{[4]}$ has evaluated the contribution of larger ionic clusters. 
In recent years, several theoretical and simulation calculations have been proposed for phase equilibria and critical properties of electrolytes ${ }^{[5-12]}$. Based on Debye-Hückel theory, Fisher and Levin $^{[7-8]}$ took into account the formation of dipolar pairs and the subsequent interaction between dipolar pairs and free ions. Using the method of associated-fluid theory, Stell and co-workers ${ }^{[9-10]}$ incorporated dipolar pairs into MSA theory. By simulation, Shelley and Patey ${ }^{[11]}$ found that the phase equilibria for an ionic fluid are similar to those for a charged hard-dumbbell fluid. Jiang et al. ${ }^{[12]}$ model an ionic fluid as a fully associated, charged hard-dumbbell fluid and use an ad hoc combination of MSA and binding-MSA ${ }^{[13]}$. Jiang's theory yields fairly good prediction of the critical temperature and critical density when compared with simulations ${ }^{[5,6]}$.

While most published theoretical work is restricted to size-symmetric electrolytes, for application to typical real ionic liquids, it is important to consider size-asymmetric ionic fluids where one ion is appreciably larger than the other. When compared with recent simulations ${ }^{[5-6]}$ for asymmetric electrolytes, the critical temperatures and critical densities calculated by MSA theory ${ }^{[14,15]}$, two theories based on Poisson-Boltzmann theory ${ }^{[14]}$ and Ebeling-Grigo theory ${ }^{[16]}$ are qualitatively incorrect. As size asymmetry rises, these theories give the wrong trend. In this work, we extend the theory of Jiang et al. ${ }^{[12]}$ to charge-symmetric but size-asymmetric ionic liquids. We report a simple theory that gives the correct trend of critical properties with size asymmetry.

\section{Theoretical Framework}

The primitive model of electrolytes concerns charged hard spheres in a continuous dielectric medium with dielectric constant $\varepsilon$. The number densities, diameters and charges of the hard 
spheres are designated by $\rho_{i}, \sigma_{i}$ and $z_{i} e$, respectively; here, $z_{i}$ is the valence and $e$ is the unit charge. The total number density of the ions is $\rho=\rho_{1}+\rho_{2}$. We consider the charge-symmetric case where $z_{1} e=-z_{2} e=z e$. The diameter ratio of the positive and negative ions, $\lambda$, gives the size asymmetry of the hard spheres:

$$
\lambda=\sigma_{1} / \sigma_{2}
$$

The collision diameter for a pair of positive and negative ions, $\sigma_{12}=\left(\sigma_{1}+\sigma_{2}\right) / 2$, provides the basic length scale appropriate for defining the reduced temperature and reduced density by

$$
\begin{aligned}
& T^{*}=k_{B} T \varepsilon \sigma_{12} / z^{2} e^{2}, \\
& \rho^{*}=\rho \sigma_{12}^{3}
\end{aligned}
$$

where $k_{B}$ is the Boltzmann constant and $T$ is the temperature.

In the theory of Jiang et al. ${ }^{[12]}$, the Helmholtz energy density $f$ has four contributions: ideal gas, hard-sphere repulsion, electrostatic interactions, and ion association:

$$
f=f^{i d}+f^{h s}+f^{e l e}+f^{a s s} .
$$

The ideal gas contribution is

$$
\beta f^{i d}=\frac{\rho}{2} \ln \frac{\rho}{2}-\frac{\rho}{2}
$$

where $\beta=1 / k_{B} T$. The hard-sphere repulsion contribution is obtained from the theoretical result of Mansoori et al. ${ }^{[17]}$ and Boublík ${ }^{[18]}$ for the equation of state of hard-sphere mixtures:

$$
\beta f^{h s}=\frac{6}{\pi}\left(\frac{\xi_{2}^{3}}{\xi_{3}^{2}}-\xi_{0}\right) \ln \left(1-\xi_{3}\right)+\frac{18 \xi_{1} \xi_{2}}{\pi\left(1-\xi_{3}\right)}+\frac{6 \xi_{2}^{3}}{\pi \xi_{3}\left(1-\xi_{3}\right)^{2}}
$$

with 


$$
\xi_{k}=\frac{\pi}{6} \sum_{i=1}^{2} \rho_{i} \sigma_{i}^{k}
$$

The electrostatic contribution is calculated by MSA theory for the asymmetric, primitive-model electrolytes $^{[2]}$ :

$$
\beta f^{e l e}=-\frac{\beta e^{2}}{\varepsilon} \sum_{i=1}^{2} \frac{\rho_{i} z_{i}}{1+\sigma_{i} \Gamma}\left[z_{i} \Gamma+\frac{\pi \sigma_{i} P_{n}}{2\left(1-\xi_{3}\right)}\right]+\frac{\Gamma^{3}}{3 \pi}
$$

with $\Gamma$ and $P_{n}$ calculated from the following two coupled equations:

$$
\begin{aligned}
& \Gamma^{2}=\frac{\pi \beta e^{2}}{\varepsilon} \sum_{i=1}^{2} \frac{\rho_{i}}{\left(1+\sigma_{i} \Gamma\right)^{2}}\left[z_{i}-\frac{\pi \sigma_{i}^{2} P_{n}}{2\left(1-\xi_{3}\right)}\right]^{2}, \\
& P_{n}=\sum_{i=1}^{2} \frac{\rho_{i} z_{i} \sigma_{i}}{1+\sigma_{i} \Gamma} /\left[1+\frac{\pi}{2\left(1-\xi_{3}\right)} \sum_{i=1}^{2} \frac{\rho_{i} \sigma_{i}^{3}}{1+\sigma_{i} \Gamma}\right] .
\end{aligned}
$$

Compared with those for the size-symmetric case, the MSA theory for the size-asymmetric case is complicated. If we approximate the asymmetric mixture by a symmetric mixture with effective diameter $\sigma_{12}$, the formulas are much simplified. Equation (8) becomes:

$$
\beta f^{e l e}=-\frac{z^{2} e^{2} \rho \beta \Gamma}{\varepsilon\left(1+\sigma_{12} \Gamma\right)}+\frac{\Gamma^{3}}{3 \pi}
$$

with $\Gamma$ calculated by

$$
\Gamma=\left[\left(1+2 \sigma_{12} \kappa\right)^{1 / 2}-1\right] / 2 \sigma_{12}
$$

where $\kappa$ is the inverse Debye screening length:

$$
\kappa^{2}=4 \pi z^{2} e^{2} \rho \beta / \varepsilon
$$

We call equations (8), (9) and (10) full-MSA, and equations (11), (12) and (13) simplified-MSA. The ion-association contribution is evaluated from first-order thermodynamic perturbation theory: 


$$
\beta f^{a s s}=-\frac{\rho}{2} \ln \left[g_{12}\left(\sigma_{12}\right) \exp \left(-\frac{z^{2} e^{2} \beta}{\varepsilon \sigma_{12}}\right)\right]
$$

where $g_{12}\left(\sigma_{12}\right)$ is the radial distribution function for the positive and negative ions at contact.

We use the exponential (EXP) approximation for $g_{12}\left(\sigma_{12}\right)^{[12]}$ :

$$
g_{12}\left(\sigma_{12}\right)=g_{12}^{\mathrm{hs}}\left(\sigma_{12}\right) \exp \left[\frac{z^{2} e^{2} \beta}{\varepsilon \sigma_{12}\left(1+\sigma_{12} \Gamma^{B}\right)^{2}}\right]
$$

where $g_{12}^{\text {hs }}\left(\sigma_{12}\right)$ is the radial distribution function for a hard-sphere mixture ${ }^{[18]}$ :

$$
g_{12}^{\text {hs }}\left(\sigma_{12}\right)=\frac{1}{1-\xi_{3}}+3\left(\frac{\sigma_{1} \sigma_{2}}{\sigma_{1}+\sigma_{2}}\right) \frac{\xi_{2}}{\left(1-\xi_{3}\right)^{2}}+2\left(\frac{\sigma_{1} \sigma_{2}}{\sigma_{1}+\sigma_{2}}\right)^{2} \frac{\xi_{2}^{2}}{\left(1-\xi_{3}\right)^{3}},
$$

and $\Gamma^{\mathrm{B}}$ is calculated from binding-MSA theory ${ }^{[13]}$ :

$$
4 \Gamma^{\mathrm{B}}\left(1+\Gamma^{\mathrm{B}} \sigma_{12}\right)^{3}=\kappa^{2} \sigma_{12} .
$$

In equations (15) and (17), we also use the assumption that an asymmetric mixture can be approximated by a symmetric mixture with effective diameter $\sigma_{12}$.

\section{Results and Discussion}

Table 1 lists reduced critical temperatures and densities for full-MSA (fMSA), simplified-MSA (sMSA) and the generalized Jiang theory based on simplified-MSA (gJiang).

Figure 1 shows reduced critical temperature and critical density as a function of size-asymmetry $\lambda$. Full-MSA gives a qualitatively incorrect trend. While the simulated critical temperature and density both decrease with rising size-asymmetry, full-MSA shows the opposite. However, simplified-MSA gives the correct trend. Taking into account the ion-association effect, the 
generalized Jiang theory based on simplified-MSA gives much better prediction. Figure 2 shows phase diagrams calculated by the generalized Jiang theory based on simplified-MSA.

Qin et al. ${ }^{[19]}$ found that for the thermodynamics properties of the size-asymmetric, dipolar hard-dumbbell fluids at high temperatures, the situation is similar: while full-MSA is qualitatively incorrect, simplified-MSA gives good prediction. Why is simplified-MSA better than the full-MSA? First, it is reasonable to approximate the asymmetric mixture by a symmetric mixture with effective diameter $\sigma_{12}$. For the asymmetric mixture, there are three length scales: $\sigma_{1}, \sigma_{2}$ and $\sigma_{12}$. Scales $\sigma_{1}$ and $\sigma_{2}$ determine interactions between similarly charged ions; however, $\sigma_{12}$ determines interactions between oppositely charged ions. As the latter contribute much more to the configurational integral than the former, to a first approximation, we can regard the three-length-scale problem as a one-length-scale problem. The thermodynamic properties are mainly determined by $\sigma_{12}$. Based on this argument, Reiss et al. ${ }^{[20]}$ proposed a corresponding-states theory for molten salts. Harvey et al. ${ }^{[21]}$ found that at reduced temperatures corresponding to aqueous electrolyte solution, simplified-MSA introduces little error when compared with MC simulations. Second, we consider the ion distribution around a fixed ion. Due to size asymmetry, there are zones around ions where only the smaller ions can exist and the larger ions are excluded. Zuckerman et al. ${ }^{[22]}$ have pointed out that it is these "charge-unbalanced" zones that lead Debye-Hückel theory to fail and give qualitatively incorrect results at low reduced temperatures. Because MSA theory is similar to Debye-Hückel theory, this argument can also explain why full-MSA fails at low temperatures. However, for simplified-MSA, where the asymmetric mixture is approximated by a symmetric mixture, the "charge-unbalanced” zones are 
excluded. This may explain why simplified-MSA gives better results.

\section{Acknowledgment}

For financial support, the authors are grateful to the Office for Basic Sciences of the US Department of Energy. 


\section{References:}

[1] P. W. Debye and E. Hückel, Phys. Z. 24, 185 (1923).

[2] L. Blum, in Theoretical Chemistry: Advances and Perspective, edited by H. Eyring and D. Henderson (Academic, New York, 1980), vol. 5, 1.

[3] W. Ebeling, Z. Phys. Chem. (Leipzig) 238, 400 (1968).

[4] M. J. Gillan, Mol. Phys. 49, 421 (1983).

[5] J. M. Romero-Enrique, G. Orkoulas, A. Z. Panagiotopoulos and M. E. Fisher, Phys. Rev. Lett. 85, 4558 (2000).

[6] Q. Yan and J. de Pable, Phys. Rev. Lett. 86, 2054 (2001).

[7] M. E. Fisher and Y. Levin, Phys. Rev. Lett. 71, 3826 (1993).

[8] Y. Levin and M. E. Fisher, Physica A 225, 164 (1996).

[9] Y. Q. Zhou, S. Yeh and G. Stell, J. Chem. Phys. 102, 5785 (1995).

[10] S. Yeh, Y. Q. Zhou and G. Stell, J. Phys. Chem. 100, 1415 (1996).

[11] J. C. Shelley and G. N. Patey, J. Chem. Phys. 103, 8299 (1995).

[12] J. Jiang, L. Blum, O. Bernard, J. M. Prausnitz and S. I. Sandler, J. Chem. Phys. 116, 7977 (2002).

[13] O. Bernard and L. Blum, J. Chem. Phys. 104, 4746 (1996).

[14] A. K. Sabir, L. B. Bhuiyan and C. W. Outhwaite, Mol. Phys. 93, 405 (1998).

[15] E. González-Tovar, Mol. Phys. 97, 1203 (1999).

[16] F. O. Raineri, J. P. Routh and G. Stell, J. Phys. IV France 10, 99 (2000).

[17] G. A. Mansoori, N. F. Carnahan, K. E. Starling and T. W. Leland, Jr., J. Chem. Phys. 54, 1523 
(1971).

[18] T. Boublík, J. Chem. Phys. 53, 471 (1970).

[19] Y. Qin, K. Zhao, H. L. Liu and Y. Hu, Mol. Simulat. 29, 743 (2003).

[20] H. Reiss, S. W. Mayer and J. L. Katz, J. Chem. Phys. 35, 820 (1961).

[21] A. H. Harvey, T. W. Copeman and J. M. Prausnitz, J Phys. Chem. 92, 6432 (1988).

[22] D. M. Zuckerman, M. E. Fisher and S. Bekiranov. Phy. Rev. E. 64, 011206 (2001). 
Table 1 Reduced critical temperatures and densities of size-asymmetric, primitive-model electrolytes calculated by full-MSA (fMSA), simplified-MSA (sMSA) and the generalized Jiang theory based on simplified-MSA (gJiang).

\begin{tabular}{|c|c|c|c|c|c|c|}
\hline \multirow{2}{*}{$\lambda$} & \multicolumn{3}{|c|}{$T_{\mathrm{c}}^{*}$} & \multicolumn{3}{|c|}{$\rho_{\mathrm{c}}{ }^{*}$} \\
\hline & fMSA & sMSA & gJiang & fMSA & sMSA & gJiang \\
\hline 1.0 & 0.0786 & 0.0786 & 0.0526 & 0.0145 & 0.0145 & 0.0645 \\
\hline 0.9 & 0.0787 & 0.0786 & 0.0526 & 0.0145 & 0.0145 & 0.0643 \\
\hline 0.8 & 0.0790 & 0.0785 & 0.0523 & 0.0147 & 0.0144 & 0.0636 \\
\hline 0.7 & 0.0796 & 0.0784 & 0.0519 & 0.0150 & 0.0142 & 0.0624 \\
\hline 0.6 & 0.0806 & 0.0782 & 0.0512 & 0.0154 & 0.0138 & 0.0604 \\
\hline 0.5 & 0.0822 & 0.0778 & 0.0502 & 0.0162 & 0.0134 & 0.0576 \\
\hline 0.4 & 0.0847 & 0.0774 & 0.0488 & 0.0174 & 0.0128 & 0.0539 \\
\hline 0.3 & 0.0887 & 0.0768 & 0.0469 & 0.0192 & 0.0120 & 0.0493 \\
\hline 0.2 & 0.0952 & 0.0760 & 0.0445 & 0.0219 & 0.0110 & 0.0439 \\
\hline 0.1 & 0.1063 & 0.0749 & 0.0416 & 0.0264 & 0.00987 & 0.0380 \\
\hline 0.05 & 0.1151 & 0.0743 & 0.0398 & 0.0299 & 0.00926 & 0.0348 \\
\hline
\end{tabular}




\section{Figure Captions}

Fig 1. Reduced critical parameters for size-asymmetric primitive-model electrolytes. (a) Reduced critical temperature as a function of size-asymmetry. (b) Reduced critical density as a function of size-asymmetry. Solid circles: Monte Carlo simulations by Yan and de Pablo ${ }^{[6]}$. Dotted lines: Calculations by full-MSA. Dashed lines: Calculations by simplified-MSA. Solid lines: Calculations by generalized Jiang theory based on simplified-MSA.

Fig. 2 Phase diagrams for size-asymmetric primitive-model electrolytes. Dots: Monte Carlo simulations by Yan and de Pablo ${ }^{[6]}$. Triangle, $\lambda=1$; Diamond, $\lambda=0.5$; Sphere, $\lambda=0.25$. Lines are calculated by Jiang theory based on simplified-MSA. 
Fig. 1(a)

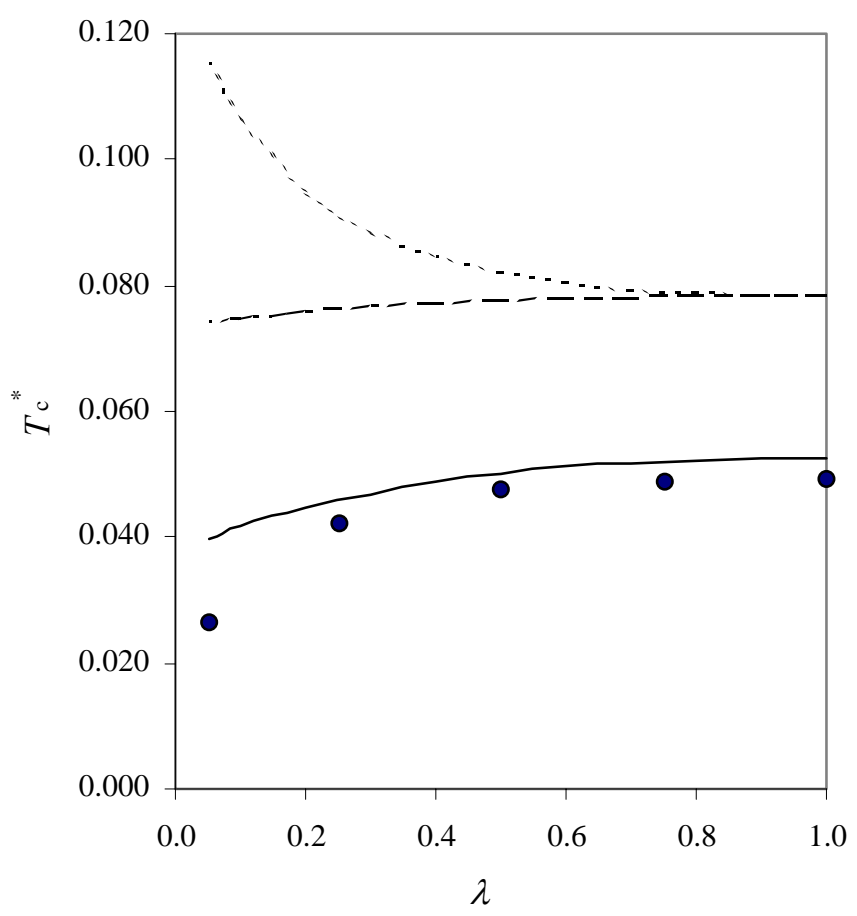


Fig. 1(b)

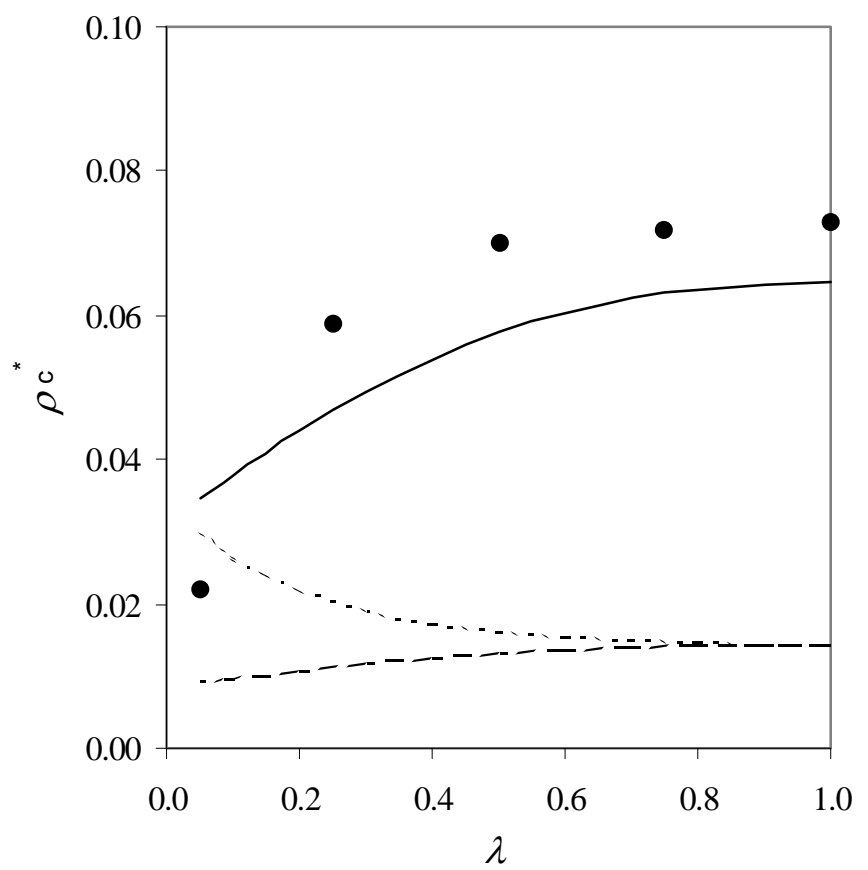


Fig. 2

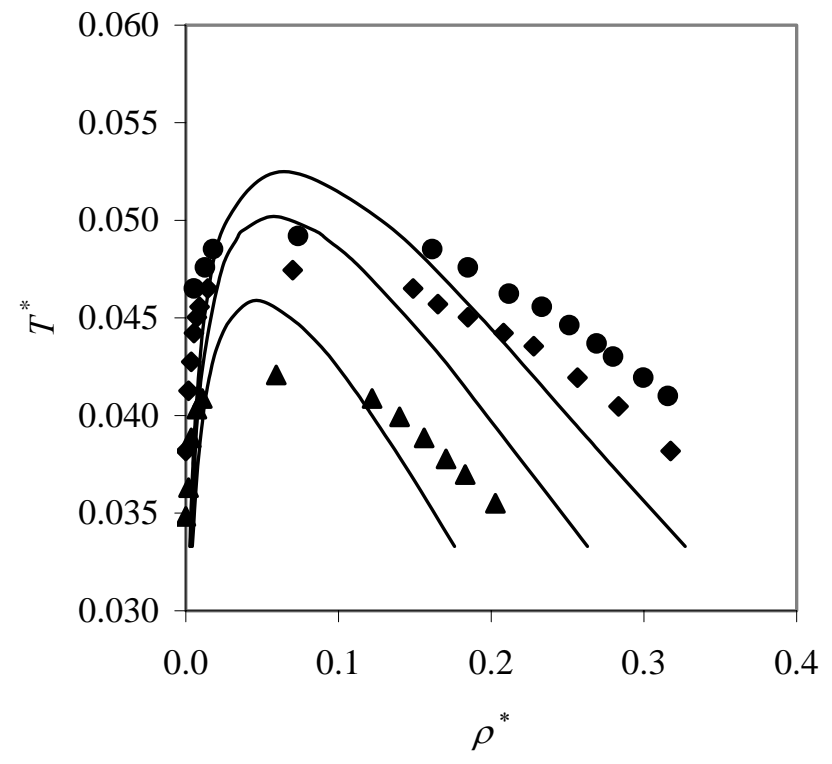

